\title{
Equivalency between the shock index and subtracting the systolic blood pressure from the heart rate: an observational cohort study
}

Yohei Kamikawa ${ }^{1 *}$ (D) and Hiroyuki Hayashi

\begin{abstract}
Background: Although the shock index is known to predict mortality and other severe outcomes, deriving it requires complex calculations. Subtracting the systolic blood pressure from the heart rate may produce a simple shock index that would be a clinically useful substitute for the shock index. In this study, we investigated whether the simple shock index was equivalent to the shock index.

Methods: This observational cohort study was conducted at 2 tertiary care hospitals. Patients who were transported by ambulance were recruited for this study and were excluded if they were aged $<15$ years, had experienced prehospital cardiopulmonary arrest, or had undergone inter-hospital transfer. Pearson's productmoment correlation coefficient and regression equation were calculated, and two one-sided tests were performed to examine their equivalency.

Results: Among 5429 eligible patients, the correlation coefficient between the shock index and simple shock index was extremely high $(0.917,95 \%$ confidence interval 0.912 to $0.921, P<.001)$. The regression equation was estimated as $\mathrm{SSI}=258.55 \mathrm{log} \mathrm{SI}$. The two one-sided tests revealed a very strong equivalency between the shock index and the index estimated by the above equation using the simple shock index (mean difference was $0.004,90 \%$ confidence interval 0.003 to 0.005 ).
\end{abstract}

Conclusion: The simple shock index strongly correlated with the shock index.

Keywords: Ambulance, Blood pressure, Cohort study, Critical illness, Heart rate, Shock index, Tertiary care hospital, Vital signs

\section{Background}

The shock index (SI) is an indicator of the severity of hypovolemic shock and is calculated by dividing the heart rate (HR) by systolic blood pressure (SBP) [1]. It serves to predict the mortality, need for blood transfusion, or necessity of intensive care unit admission

\footnotetext{
* Correspondence: fundarike ykami@yahoo.co.jp

'Department of Emergency Medicine, University of Fukui Hospital, 23-3 Matsuoka Shimoaizuki, Eiheiji-cho, Yoshida-gun, Fukui 910-1193, Japan Full list of author information is available at the end of the article
}

among patients with trauma [2-7], postpartum haemorrhage $[8,9]$, acute myocardial infarction $[10,11]$, stroke $[12,13]$, sepsis $[14,15]$, and other critical conditions $[16$, 17]. Numerous previous studies have demonstrated that the SI demonstrates superior prediction for mortality to traditional vital signs, although it has some limitations, including its low sensitivity especially for the elderly or obstetric patients [2-17]. However, in clinical practice, calculating the SI for all patients is difficult. An SI value > 0.9 is generally accepted as a cut-off point for an increased

(c) The Author(s). 2020 Open Access This article is licensed under a Creative Commons Attribution 4.0 International License, which permits use, sharing, adaptation, distribution and reproduction in any medium or format, as long as you give appropriate credit to the original author(s) and the source, provide a link to the Creative Commons licence, and indicate if changes were made. The images or other third party material in this article are included in the article's Creative Commons licence, unless indicated otherwise in a credit line to the material. If material is not included in the article's Creative Commons licence and your intended use is not permitted by statutory regulation or exceeds the permitted use, you will need to obtain permission directly from the copyright holder. To view a copy of this licence, visit http://creativecommons.org/licenses/by/4.0/. The Creative Commons Public Domain Dedication waiver (http://creativecommons.org/publicdomain/zero/1.0/) applies to the data made available in this article, unless otherwise stated in a credit line to the data. 
risk of mortality [16], but it is sometimes difficult to quickly calculate whether the patient meets this cut-off when the value of the quotient (particularly when considering the second decimal place) is extremely close to 0.9 (e.g. When a patient has an HR of 103 beats per minute and SBP of $114 \mathrm{mmHg}$, the quotient is approximately 0.904 and it technically meets the cut-off but it is exceedingly difficult to calculate promptly without a calculator). Having a confusing cut-off value, the SI needs to be interpreted from several variables to identify patients with a critical status but stable HR and SBP making it an impractical indicator that is rarely used in scoring systems assessing emergencies. Furthermore, despite its utility, there is no established consensus on when and where to utilize SI in emergency departments (EDs) [18]. Recent studies have attempted to validate revised SI measurements meant to improve its ability to predict mortality [19-22]; however, such calculations are more complicated and tend to be avoided by clinicians. If the calculation of the SI can be made simpler, it would lead to rapid progress in terms of the clinical research using SI.

Considering that SI is used to represent the different dynamics of HR and SBP [23, 24], it is possible that simply subtracting the SBP from the HR may provide a useful substitute for SI, improving the availability of a calculated value as it is easier to mentally subtract integers than to divide them.

In this study, we identify the proposed new index the simple shock index (sSI) and investigated whether the sSI predicted SI equivalently among patients transported to hospitals via ambulance.

\section{Methods}

\section{Study design and setting}

This observational cohort study was conducted at two urban tertiary hospitals that annually receive via ambulance transport patients ( $>2500$ and $>4000$ respectively). Written informed consent was waived because of the retrospective observational nature of the study, which was conducted using the opt-out method on the hospital websites. All data were fully anonymized. The institutional ethical review board of the University of Fukui Hospital (20160131) and the Fukui Prefectural Hospital (16-60) approved the study's protocol. All methods were carried out in accordance with relevant guidelines and regulations.

Patients were considered eligible if they were transported to either hospital via ambulance between July 1 , 2015 and June 30, 2016. Patients who were aged $<15$ years, experienced prehospital cardiopulmonary arrest, or underwent inter-hospital transfer were excluded.

\section{Study protocol}

The collected data included HR in the ED, SBP in the $\mathrm{ED}$, age, sex, trauma, pregnancy status, acute myocardial infarction, sepsis, chronic respiratory disease (previous history of chronic obstructive pulmonary disease, chronic bronchitis, asthma, bronchiectasis, interstitial pneumonia, pulmonary tuberculosis, or lung cancer), and intracranial disease (having suffered from stroke, transient ischemic attack, encephalitis, encephalopathy, seizure, brain tumour, hydrocephalus, concussion, cerebral contusion, or traumatic subarachnoid haemorrhage at arrival to ED). These specific patient characteristics were included since many previous studies have examined the ability of the SI to predict mortality or other critical conditions in those with trauma, pregnancy, acute myocardial infarction, sepsis, and intracranial disease [2-15], and because HR and SBP of aged or chronic respiratory disease patients are known to exhibit specific dynamics [25-27].

HR and SBP were documented immediately following a patient's arrival to ED. These data were extracted from the electronic medical records. When available, prehospital vital signs documented in emergency service records were used to substitute for missing ED vital sign data. The bedside monitor models BSM-3562 (NIHON KOHDEN, Tokyo, Japan) and PVM-2703 (NIHON KOHDEN, Tokyo, Japan) were used to measure prehospital and in-hospital vital signs, respectively. Any remaining missing data were complemented using the multiple imputation method $[28,29]$. To minimize selection or operator bias, all data were collected retrospectively and were fully anonymized before analysis.

The SI and sSI were calculated from the HR and SBP as mentioned above $(\mathrm{SI}=\mathrm{HR} / \mathrm{SBP} ; \mathrm{sSI}=\mathrm{HR}-\mathrm{SBP})$.

\section{Statistical analysis}

Categorical variables were reported as numbers and percentages, while continuous variables were reported as the median and interquartile range (IQR). Patients aged 65 years and older were classified as aged individuals according to the definition widely adopted in developed countries [30]. We used a generally accepted consensus and the 2018 European Society of Cardiology/European Society of Hypertension Guidelines for the management of arterial hypertension [31,32] to classify patients into four categories according to their SBP as follows: those with SBP of $<90 \mathrm{mmHg}$ were included in the hypotension group, those with SBP of $90-139 \mathrm{mmHg}$ were included in the normotension group, those with SBP of $140-179 \mathrm{mmHg}$ were included in the hypertension group, and those with SBP $\geq 180 \mathrm{mmHg}$ were included in the severe hypertension group.

First, a correlation plot for SI and sSI derived from all subjects was constructed, and the Pearson's productmoment correlation coefficient was calculated. Regression analysis was performed using the least-squares method where possible. According to the regression 
equation, the sSI value, which corresponds to the SI value of 0.9 , was determined.

Next, an equivalence test with two one-sided test (TOST) was used to examine the mean difference between the SI and estimated value of SI derived by sSI from the regression equation mentioned above. It was necessary to convert sSI to the same scale as SI using the procedure noted previously since TOST compares mean difference between two groups. In a TOST, equivalency is determined when the $90 \%$ confidence interval (CI) of mean difference is settled within a predetermined equivalence margin [33]. Since the equivalence margin between 0.25 and 0.5 of effect size adjusted for standard deviation is usually chosen in practice [34], we chose 0.25 of the standardized effect size of the SI as the equivalent margin. Power values of 0.8 were considered statistically significant. Equivalence tests were also performed for 14 patient subgroups including aged, nonaged, female, male, trauma, pregnant, acute myocardial infarction, sepsis, chronic respiratory disease, intracranial disease, hypotension, normotension, hypertension, and severe hypertension. The potential risk of type I errors due to multiple subgroup analyses would be expected to occur in up to 0.7 nominally statistically significant interaction tests $(P<0.05)$ by chance alone, which was a sufficiently low possibility [35].

Finally, we performed a sensitivity analysis to validate robustness with respect to missing data with an equivalence test using all cases that were not missing any data [36]. $\mathrm{R}$ software, version 3.4.1 (The $\mathrm{R}$ Foundation, Vienna, Austria) was used for all statistical analyses.

\section{Results}

\section{Characteristics of the study subjects}

There were 6687 patients who were transported to the two hospitals via ambulance during the study period;
1258 of these patients were excluded including 527 aged $<15$ years, 136 who experienced prehospital cardiopulmonary arrest, and 595 who underwent inter-hospital transfer. Thus, 5429 patients were ultimately evaluated (Fig. 1). The patients' characteristics are shown in Table 1. The median age was 68 (IQR 47-81), and the median ages of those aged $15-64$ and aged $\geq 65$ were 43 (IQR 29-56) and 80 (IQR 73-85), respectively. The median HR and SBP values were 84 (IQR 72-97) and 140 (IQR 121-163) mmHg, respectively. Prehospital HR of 765 cases (14.1\%) and prehospital SBP of 721 cases (13.3\%) were substituted for missing values of the ED. Remaining missing HR and SBP values were complemented by using the multiple imputation method for $958(17.6 \%)$ and $912(16.8 \%)$ patients, respectively. No other data were missing.

\section{Main results}

The Pearson's product-moment correlation coefficient between the SI and sSI was 0.917 (95\% CI 0.912-0.921, $P<.001$ ), indicating an extremely high correlation. The $\log \mathrm{SI} / \mathrm{sSI}$ correlation plot represented a proportional relationship (Fig. 2) and the regression equation was estimated as $\mathrm{sSI}=258.55 \log$ SI using the least-squares method with logarithmic transformation. According to this equation, an sSI value of -12 was found to correspond to the SI value of 0.9 .

Next, an equivalence test with TOST was performed for comparisons between SI and the estimated value of SI derived by SSI. The estimated value of SI was calculated as $10^{\mathrm{sSI} / 258.55}$ according to the regression equation mentioned above. Equivalence margin was determined as \pm 0.052 , since it was 0.25 of the standardized effect size of SI. The TOST revealed an equivalency between the SI and sSI (mean difference, 0.004; 90\% CI, 0.003 to 0.005 ; statistical power, $100.00 \%)$. Similar consequences

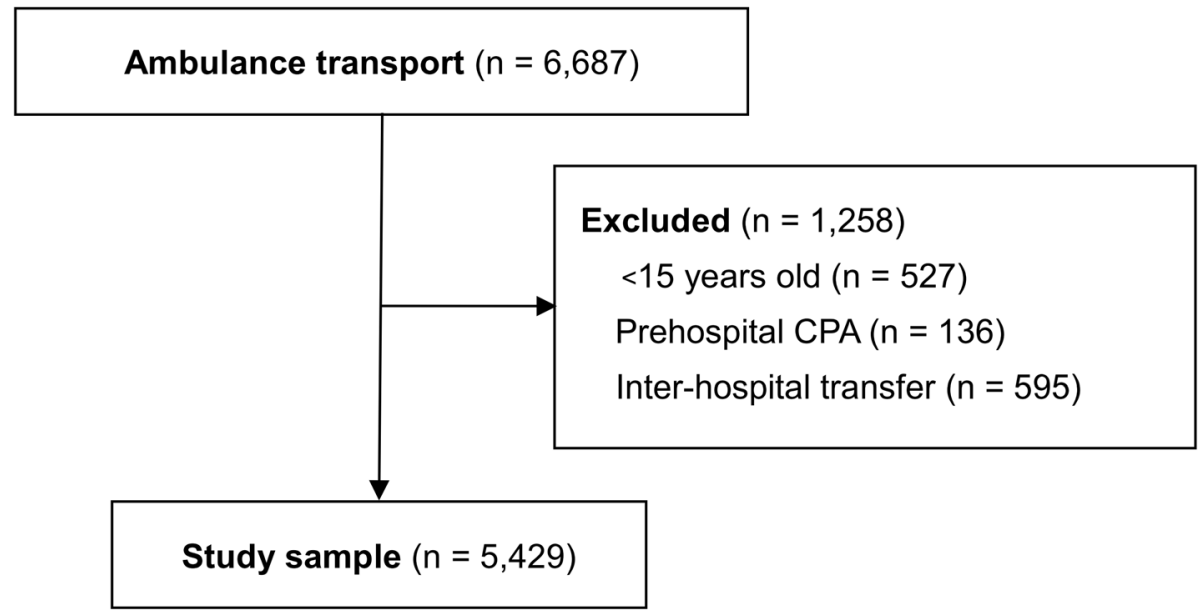

Fig. 1 Flowchart of the study 
Table 1 Patients' Characteristics

\begin{tabular}{ll}
\hline & N (\%) \\
\hline Age, years & \\
$15-64$ & $2420(44.6)$ \\
$\geq 65$ & $3009(55.4)$ \\
Sex & \\
Female & $2677(49.3)$ \\
Male & $2752(50.7)$ \\
Pre-existing condition & \\
Trauma & $1653(30.4)$ \\
Pregnancy & $91(1.7)$ \\
Acute myocardial infarction & $97(1.8)$ \\
Sepsis & $65(1.2)$ \\
Chronic respiratory disease & $114(2.1)$ \\
Intracranial disease & $643(11.8)$ \\
Systolic blood pressure & \\
Hypotension (<90 mmHg) & $129(23.8)$ \\
Normotension (90-139 mmHg) & $2467(45.4)$ \\
Hypertension (140-179 mmHg) & $2282(42.0)$ \\
Severe hypertension $(\geq 180 \mathrm{mmHg})$ & $551(10.1)$ \\
\hline
\end{tabular}

were also derived from subgroup analyses except for severe hypertension (Table 2, Fig. 3). The statistical power for all analyses of each subgroup and all subjects were over $97.29 \%$.

In the sensitivity analysis, the robustness of equivalency was validated using 3629 cases $(66.8 \%)$ that did not lack any data of ED (mean difference was $0.006,90 \%$ CI 0.004 to 0.007 , statistical power $100.00 \%$ ).

\section{Discussion}

In this study, we revealed that the sSI, which was derived by subtracting the SBP from the HR, strongly correlated with the SI among the patients transported via ambulance. Meanwhile, an sSI value of $>-12$ was observed to correspond to the known SI cut-off value of $>0.9$, which is the most common optimized cut-off point, as has been previously described [16]. However, as positive numbers are more easily calculated and interpreted, instead of calculating the sSI cut-off using $\mathrm{HR}-\mathrm{SBP}>-12$ as we have done in the present study, we recommend using either SBP $-\mathrm{HR}<12$ or HR $+12>\mathrm{SBP}$ instead, which can be considered to be equivalent to an $\mathrm{SI}>0.9$.

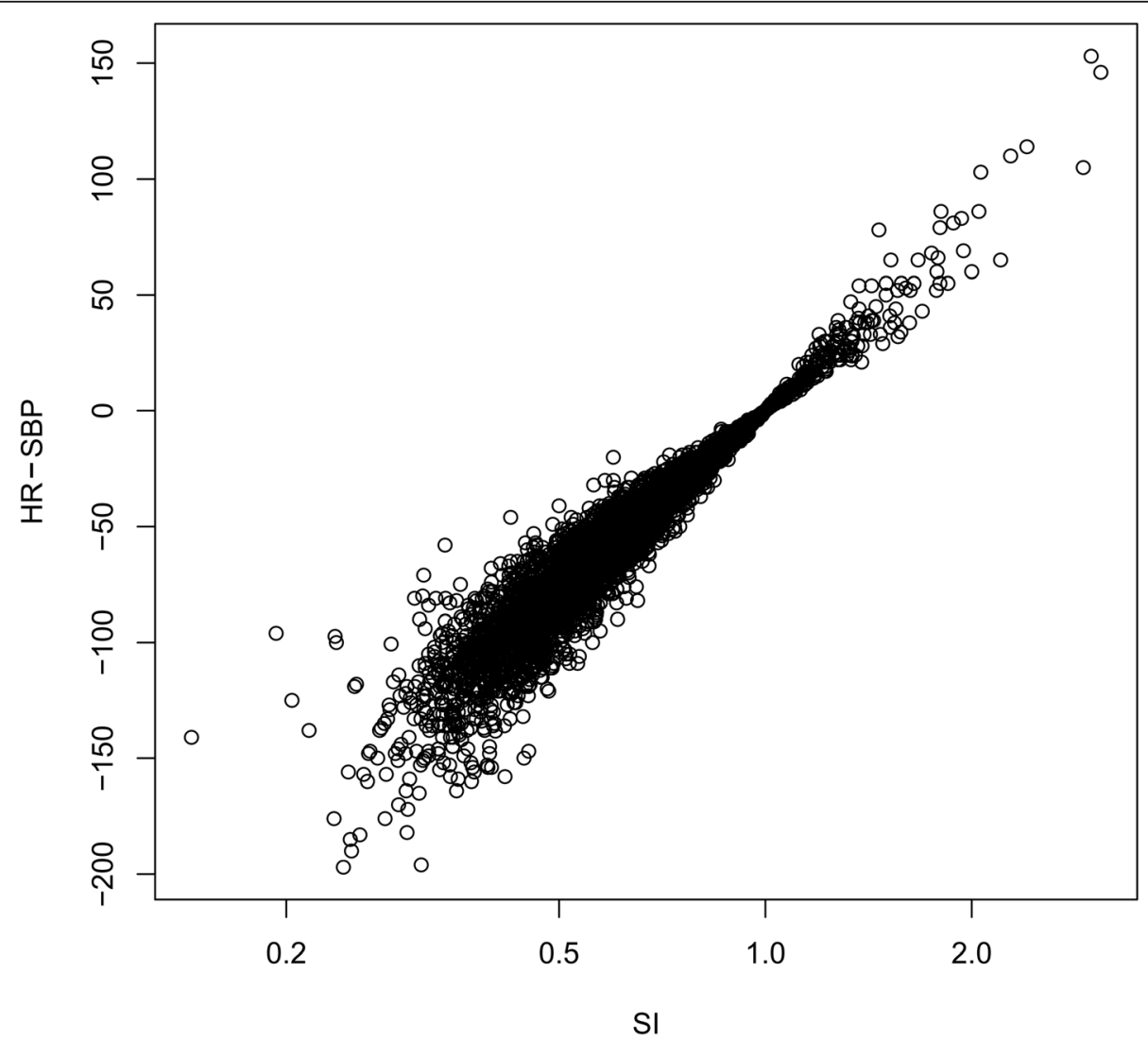

Fig. 2 Correlation plot of the SI and SSI (HR - SBP). SI: shock index, sSI: simple shock index, HR: heart rate, SBP: systolic blood pressure. SI plotted on logarithmic scale 
Table 2 SI and SI derived by SSI (HR-SBP) equivalence tests

\begin{tabular}{|c|c|c|}
\hline & $\mathrm{N}$ & Mean difference $(90 \% \mathrm{Cl})$ \\
\hline \multicolumn{3}{|l|}{ Age, years } \\
\hline $15-64$ & 2420 & $0.009(0.008 \text { to } 0.011)^{*}$ \\
\hline$\geq 65$ & 3009 & $0.000(-0.001 \text { to } 0.002)^{*}$ \\
\hline \multicolumn{3}{|l|}{ Sex } \\
\hline Female & 2677 & $0.003(0.001 \text { to } 0.004)^{*}$ \\
\hline Male & 2752 & $0.005(0.004 \text { to } 0.007)^{*}$ \\
\hline \multicolumn{3}{|l|}{ Pre-existing condition } \\
\hline Trauma & 1653 & $0.000(-0.002 \text { to } 0.002)^{*}$ \\
\hline Pregnancy & 91 & $0.008(0.004 \text { to } 0.012)^{*}$ \\
\hline Acute myocardial infarction & 97 & $0.016(0.006 \text { to } 0.026)^{*}$ \\
\hline Sepsis & 65 & $-0.022(-0.038 \text { to }-0.006)^{*}$ \\
\hline Chronic respiratory disease & 114 & $-0.003(-0.011 \text { to } 0.005)^{*}$ \\
\hline Intracranial disease & 643 & $-0.005(-0.008 \text { to }-0.002)^{*}$ \\
\hline \multicolumn{3}{|l|}{ Systolic blood pressure } \\
\hline Hypotension (<90 mmHg) & 129 & $0.008(-0.014 \text { to } 0.031)^{*}$ \\
\hline Normotension (90-139 mmHg) & 2467 & $0.030(0.029 \text { to } 0.031)^{*}$ \\
\hline Hypertension (140-179 mmHg) & 2282 & $-0.007(-0.009 \text { to }-0.006)^{*}$ \\
\hline Severe hypertension ( $\geq 180 \mathrm{mmHg}$ ) & 551 & $-0.064(-0.066$ to -0.061$)$ \\
\hline Total & 5429 & $0.004(0.003 \text { to } 0.005)^{*}$ \\
\hline
\end{tabular}

SI Shock index, sSI Simple shock index, HR Heart rate, SBP Systolic blood pressure, CI Confidence interval. Equivalence margin is 0.052 . SI derived by sSI was calculated according to the following estimation equation: $\mathrm{sSI}=258.55 \mathrm{log} \mathrm{SI}$.

*Significant results based on the $90 \% \mathrm{Cl}$ values

This finding confirms the utility of using the sSI for more rapid assessment of the condition of patients admitted for emergency care than the more complicated SI. Assuming that a calculator is not available, judging whether the SI is more than 1.0 is quite easy because the hypothesis is true when the value of HR is greater than that of SBP. However, when the SI cut-off value is 0.9, the judgement becomes difficult. As calculating HR/SBP mentally can cause confusion, we often calculate SBP times 0.9 mentally and then compare it with HR. For example, when an HR of 103 and SBP of 114 are known, we calculate $114 \times 0.9=102.6$ and then compare it with 103. Thus, we can judge SI to be $>0.9$ because $103>$ 102.6. Obviously, this procedure is complicated and tends to lead to miscalculation. On the other hand, an alternative criterion of $\mathrm{sSI}>-12$ makes the procedure much simpler. For example, using the same values of HR of 103 and SBP of 114, calculating HR plus 12 is the first step to solve the hypothesis. When the sum (e.g. $103+12=115)$ is compared with SBP, we are able to judge that sSI is $>-12$ because $115>114$. Since this addition is quite easy, mental calculation can be performed at a glance.

While attempts have been made to improve the predictive ability of SI for mortality or other outcomes, these endeavours have made the process more complicated. Examples of such previously proposed predictors include an index called 'age shock index' derived by multiplying the SI with the patient's age, or another referred to as the 'modified shock index' obtained by dividing the HR by the mean blood pressure $[19,20]$. Other complicated predictors were also proposed such as 'respiratory adjusted shock index' calculated by multiplying the SI with the respiratory rate/10 and 'reverse shock index multiplied by Glasgow Coma Scale score' derived by dividing the Glasgow Coma Scale by the SI $[21,22]$. To the best of our knowledge, this is the first study aimed at simplifying the calculation using subtraction, as no previous groups have proposed the idea of subtracting the SBP from the HR for purposes of estimating the SI.

The sSI was shown to be equivalent to SI in most subgroups by TOST and only significantly underestimated SI in the severe hypertension subgroup. However, we believe this finding will have minimal on the utility of sSI in clinical practice mainly because SI was mainly developed to identify patients who were in a critical state despite their vital signs being within normal range $[16,31]$. Another reason is that very low SI values should not be ignored as they can often pose risks to patients contrary to what is expected. Several studies have reported a Jshaped relationship between SI and mortality [4, 13], 


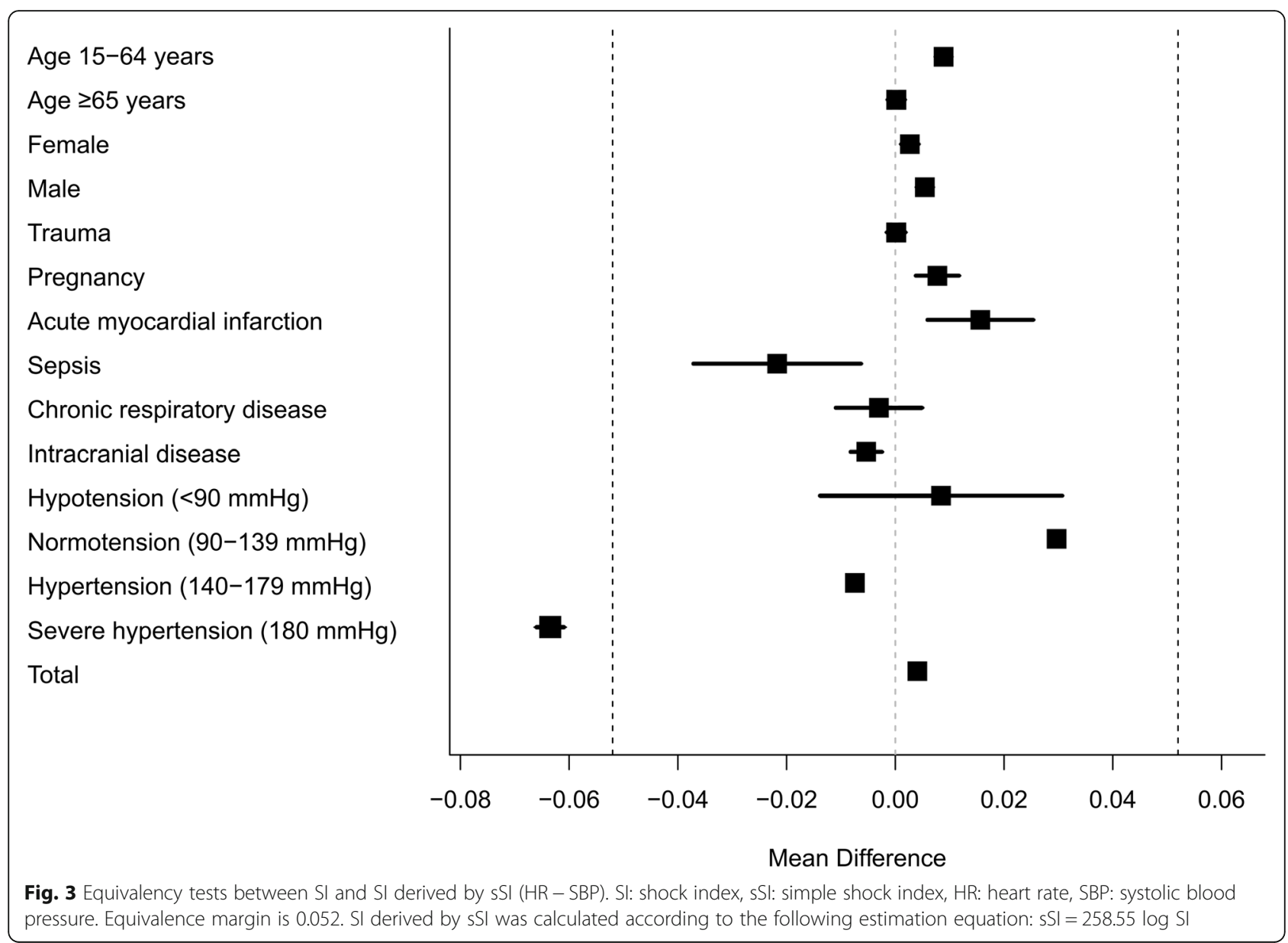

that is mortality increases when the SI value is under 0.5. Therefore, our new indicator sSI would not be utilized on patients with severe hypertension who would typically require close monitoring as their SI values tend to be very low.

Regarding the moderate number of missing values, when comparing analysis using values generated from the imputation method and from using only cases without missing values our results indicated good concordance between the measures examined and indicated that $\mathrm{sSI}$ is a useful and precise tool.

This study has several limitations. We were unable to investigate patients of different ethnicities because this study was conducted in a single geographic area. Moreover, there is a dearth of a theoretical framework to support the sSI, given that this study was intended as merely a proposal of a pragmatic alternative to the SI. Additionally, although the SI is used to predict mortality, necessity of blood transfusion, or necessity for intensive care unit admission, our study did not address these outcomes; we tested only the correlation between the SI and sSI here. Moreover, we could not prove that the assessed patients were clinically in shock or not since this diagnosis is determined using various factors such as cardiac output, lactate level, urine output, blood gas analyses, and mental status. Furthermore, there is certainly a possibility of multiplicity in the subgroup analyses, although this possibility was estimated to be sufficiently low. These issues should be addressed in future studies intended to clarify the scientific underpinnings of sSI, to further validate $\mathrm{sSI}$ as an accurate substitute calculation for SI, or to justify the clinical utility of sSI.

\section{Conclusions}

The sSI was demonstrated to highly correlate with the SI among patients transported to hospitals in our study via ambulance, though further studies are needed to validate its clinical utility. Given that the sSI is easier to calculate and use for performing evaluations, it can be a useful and highly precise alternative to the SI.

\section{Abbreviations}

SI: Shock index; HR: Heart rate; SBP: Systolic blood pressure; sSI: Simple shock index; ED: Emergency department; IQR: Interquartile range; TOST: Two onesided test; Cl: Confidence interval 


\section{Acknowledgements}

We would like to thank Editage (www.editage.jp) for English language editing.

\section{Authors' contributions}

YK conceived the study, designed the trial, collected data, handled the recruitment of patients, managed the data including quality control, analysed the data, and drafted the manuscript. HH supervised the conduct of the trial, provided statistical advice on study design, and chaired the data oversight committee. All authors read and approved the final manuscript.

\section{Funding}

This research received no specific grant from any funding agency in the public, commercial or not-for-profit sectors.

\section{Availability of data and materials}

The datasets generated and/or analysed during the current study are available in the Open Science Framework repository, https://osfio/n94zs/ or DOI https://doi.org/10.17605/OSF.IO/N94ZS.

\section{Ethics approval and consent to participate}

This study's protocol was approved by the research ethics committee of the University of Fukui Hospital (20160131) and the Fukui Prefectural Hospital (16-60). Informed consent was not required because the information was sufficiently anonymized.

\section{Consent for publication}

Not applicable.

\section{Competing interests}

The authors declare that they have no competing interests.

\section{Author details}

'Department of Emergency Medicine, University of Fukui Hospital, 23-3 Matsuoka Shimoaizuki, Eiheiji-cho, Yoshida-gun, Fukui 910-1193, Japan. ${ }^{2}$ Department of General Medicine, University of Fukui Hospital, 23-3 Matsuoka Shimoaizuki, Eiheiji-cho, Yoshida-gun, Fukui 910-1193, Japan.

\section{Received: 10 September 2020 Accepted: 27 October 2020}

\section{Published online: 31 October 2020}

\section{References}

1. Allgower M, Burri C. Shock index. Dtsch Med Wochenschr. 1967;92:1947-50.

2. Sloan EP, Koenigsberg M, Clark JM, Weir WB, Philbin N. Shock index and prediction of traumatic hemorrhagic shock 28-day mortality: data from the DCLHb resuscitation clinical trials. West J Emerg Med. 2014;15:795-802.

3. Cannon CM, Braxton CC, Kling-Smith M, Mahnken JD, Carlton E, Moncure M. Utility of the shock index in predicting mortality in traumatically injured patients. J Trauma. 2009;67:1426-30

4. Odom SR, Howell MD, Gupta A, Silva G, Cook CH, Talmor D. Extremes of shock index predicts death in trauma patients. J Emerg Trauma Shock. 2016;9:103-6.

5. Schroll R, Swift D, Tatum D, Couch S, Heaney JB, Llado-Farrulla M, et al. Accuracy of shock index versus $A B C$ score to predict need for massive transfusion in trauma patients. Injury. 2018;49:15-9

6. McNab A, Burns B, Bhullar I, Chesire D, Kerwin A. A prehospital shock index for trauma correlates with measures of hospital resource use and mortality. Surgery. 2012;152:473-6.

7. Bruijns SR, Guly HR, Bouamra O, Lecky F, Wallis LA. The value of the difference between ED and prehospital vital signs in predicting outcome in trauma. Emerg Med J. 2014;31:579-82.

8. Nathan HL, AEI A, Hezelgrave NL, Seed P, Butrick E, Miller S, et al. Shock index: an effective predictor of outcome in postpartum haemorrhage? BJOG. 2015:122:268-75.

9. Nathan HN, Seed PT, Hezelgrave NL, Greeff AD, Lawley E, Anthony J, et al. Shock index thresholds to predict adverse outcomes in maternal hemorrhage and sepsis: a prospective cohort study. Acta Obstet Gynecol Scand. 2019;98:1178-86.

10. Abe N, Miura T, Miyashita Y, Hashizume N, Ebisawa S, Motoki H, et al. Longterm prognostic implications of the admission shock index in patients with acute myocardial infarction who received percutaneous coronary intervention. Angiology. 2017;68:339-45.
11. Wang Q, Shen H, Mao H, Yu F, Wang H, Zheng J. Shock index on admission is associated with coronary slow/no reflow in patients with acute myocardial infarction undergoing emergent percutaneous coronary intervention. J Interv Cardiol. 2019;2019:7873468.

12. McCall SJ, Musgrave SD, Potter JF, Hale R, Clark AB, Mamas MA, et al. The shock index predicts acute mortality outcomes in stroke. Int J Cardiol. 2015;182:523-7.

13. Myint PK, Sheng S, Xian Y, Matsouaka RA, Reeves MJ, Saver JL, et al. Shock index predicts patient-related clinical outcomes in stroke. J Am Heart Assoc. 2018;7:e007581.

14. Berger T, Green J, Horeczko T, Hagar Y, Garg N, Suarez A, et al. Shock index and early recognition of sepsis in the emergency department: pilot study. West J Emerg Med. 2013;14:168-74.

15. Middleton DJ, Smith TO, Bedford R, Neilly M, Myint PK. Shock index predicts outcome in patients with suspected sepsis or community-acquired pneumonia: a systematic review. J Clin Med. 2019:8:1144.

16. Rady MY, Smithline HA, Blake H, Nowak R, Rivers E. A comparison of the shock index and conventional vital signs to identify acute, critical illness in the emergency department. Ann Emerg Med. 1994;24:685-90.

17. Kamikawa $Y$, Hayashi $H$. Predicting in-hospital mortality among non-trauma patients based on vital sign changes between prehospital and in-hospital: an observational cohort study. PLoS One. 2019:14:e0211580.

18. Koch E, Lovett S, Nghiem T, Riggs RA, Rech MA. Shock index in the emergency department: utility and limitations. Open Access Emerg Med. 2019;11:179-99.

19. Zarzaur BL, Croce MA, Magnotti LJ, Fabian TC. Identifying life-threatening shock in the older injured patient: an analysis of the National Trauma Data Bank. J Trauma. 2010;68:1134-8.

20. Liu Y, Liu J, Fang ZA, Shan G, Xu J, Qi Z, et al. Modified shock index and mortality rate of emergency patients. World J Emerg Med. 2012;3:114-7.

21. Caputo N, Reilly J, Kanter M, West J. A retrospective analysis of the respiratory adjusted shock index to determine the presence of occult shock in trauma patients. J Trauma Acute Care Surg. 2018:84:674-8.

22. Kimura A, Tanaka N. Reverse shock index multiplied by Glasgow coma scale score $(\mathrm{rSIG})$ is a simple measure with high discriminant ability for mortality risk in trauma patients: an analysis of the Japan trauma data Bank. Crit Care. 2018;22:87.

23. Graham LN, Smith PA, Stoker JB, Mackintosh AF, Mary DA. Sympathetic neural hyperactivity and its normalization following unstable angina and acute myocardial infarction. Clin Sci. 2004;106:605-11.

24. Barcroft H, Edholm OG, McMichael J, Sharpey-Schafer EP. Posthaemorrhagic fainting: study by cardiac output and forearm flow. Lancet. 1944;243:489-91.

25. Lamantia MA, Stewart PW, Platts-Mills TF, Biese KJ, Forbach C, Zamora E, et al. Predictive value of initial triage vital signs for critically ill older adults. West J Emerg Med. 2013;14:453-60.

26. Chester JG, Rudolph JL. Vital signs in older patients: age-related changes. J Am Med Dir Assoc. 2011;12:337-43.

27. Eccles SR, Subbe C, Hancock D, Thomson N. CREWS: improving specificity whilst maintaining sensitivity of the National Early Warning Score in patients with chronic hypoxaemia. Resuscitation. 2014;85:109-11.

28. Haukoos JS, Newgard CD. Advanced statistics: missing data in clinical research - part 1: an introduction and conceptual framework. Acad Emerg Med. 2007;14:662-8.

29. Newgard CD, Haukoos JS. Advanced statistics: missing data in clinical research —part 2: multiple imputation. Acad Emerg Med. 2007:14:669-78.

30. World Health Organization. Proposed working definition of an older person in Africa for the MDS Project. 2002. http://www.who.int/healthinfo/survey/ ageingdefnolder/en/ Accessed 15 October 2020

31. Vandromme MJ, Griffin RL, Kerby JD, McGwin G Jr, Rue LW 3rd, Weinberg JA. Identifying risk for massive transfusion in the relatively normotensive patient: utility of the prehospital shock index. J Trauma. 2011;70:384-90

32. Williams B, Mancia G, Spiering W, Rosei EA, Azizi M, Burnier M, et al. 2018 ESC/ESH guidelines for the management of arterial hypertension: the task force for the management of arterial hypertension of the European Society of Cardiology (ESC) and the European Society of Hypertension (ESH). Eur Heart J. 2018;39:3021-104.

33. Walker $E$, Nowacki AS. Understanding equivalence and noninferiority testing. J Gen Intern Med. 2010;26:192-6.

34. Chow S, Shao J, Wang H, Lokhnygina Y. Sample size calculations in clinical research. 3rd ed. Boca Raton: CRC Press; 2017.

35. Wang R, Lagakos SW, Ware JH, Hunter DJ, Drazen JM. Statistics in medicine — reporting of subgroup analyses in clinical trials. N Engl J Med. 2007;357: 2189-94. 
36. Thabane L, Mbuagbaw L, Zhang S, Samaan Z, Marcucci M, Ye C, et al. A tutorial on sensitivity analyses in clinical trials: the what, why, when and how. BMC Med Res Methodol. 2013;13:92.

\section{Publisher's Note}

Springer Nature remains neutral with regard to jurisdictional claims in published maps and institutional affiliations.

Ready to submit your research? Choose BMC and benefit from:

- fast, convenient online submission

- thorough peer review by experienced researchers in your field

- rapid publication on acceptance

- support for research data, including large and complex data types

- gold Open Access which fosters wider collaboration and increased citations

- maximum visibility for your research: over $100 \mathrm{M}$ website views per year

At BMC, research is always in progress. 